lungen zum politischen System. Aus methodischer Sicht sind die umfangreichen Ergebnisse, die hier im Einzelnen nicht ausgeführt werden können, aber nicht unproblematisch. Die Autoren verweisen auf das Problem der Unterschätzung des Nichtwähleranteils bei Umfragen durch systematische Falschangaben und Ausfälle. Sie bemerken sogar Zykluseffekte in Umfragen innerhalb der Wahlperioden, die nicht von der Erinnerungsleistung der Befragten abzuhängen scheinen. Darüber hinaus ist es äußerst fragwürdig, mit Regressionsparametern von Querschnittsdaten Trendentwicklungen erklären zu wollen.

Steinbrecher, Huber und Rattinger zeigen, dass die Erklärung der Wahlbeteiligung in ihren vielen Dimensionen nicht trivial ist. Die Bewertung der Ergebnisse - Krise oder Normalisierung - hängt letztlich vom normativen Standpunkt des Betrachters ab.

"Turnout in Germany“ ist eine Arbeit in der mehr als nur die Standardwerkzeuge der Wahlanalyse zum Einsatz kommen und wichtige Erkenntnisse um die Wahlbeteiligung und das individuelle Nichtwählen gewonnen und reproduziert werden. Das Buch spiegelt den aktuellen Stand der quantitativen Nichtwähler- und Wahlbeteiligungsforschung in Deutschland wider und wird auf absehbare Zeit das Standardwerk zu diesem Thema sein.

Ossip Fürnberg

\title{
Gefüllte Lücke in der deutschen Wahl- und Einstellungsforschung
}

Bytzek, Evelyn: Ereignisse und ihre Wirkung auf die Popularität von Regierungen. Von der Schleyer-Entführung zur Elbeflut (Studien zur Wahl-und Einstellungsforschung, Band 6), Nomos Verlagsgesellschaft, Baden-Baden 2007, 229 Seiten, € 34,90.

Evelyn Bytzek stellt sich in ihrer Dissertation dem Problem, wie politische Ereignisse auf die Popularität und damit auch die Wiederwahlchancen von Regierungen wirken. Dabei geht es zum einen um den bekannten Topos, dass Krisenzeiten Regierungszeiten wären. Das Elbehochwasser im Jahre 2002, das nach gängiger Interpretation Gerhard Schröder zur Wiederwahl verhalf, kann hier als paradigmatischer Fall gelten. Zum anderen stehen Skandale wie die CDU-Spendenaffäre 1999/2000, die Flick- und die Spiegelaffäre im Mittelpunkt sowie weitere Ereignistypen. Bytzek möchte herausfinden, welche Klassen von Ereignissen tatsächlich Popularität steigern, was genau die Wirkungsmechanismen sind sowie welcher Art und Dauer diese Wirkung ist. Ereignisse sollen also nicht ad-hoc analysiert werden, wie bislang üblich, wenn andere Erklärungen versagten, sondern systematisch und mit Blick auf regelmäßige Effekte.

Bei ihrem Vorhaben kann Bytzek an eine ausführlich in den USA geführte Debatte anknüpfen. Die dortige Situation könne, so die Autorin, jedoch nur in begrenztem Umfang auf die Bundesrepublik Deutschland übertragen werden. Zu unterschiedlich sei die Struktur der politischen Systeme und als Konsequenz daraus des Parteienwettbewerbs. Übertragbar ist jedoch die Feststellung, dass außerordentliche Ereignisse die Handlungsfähigkeit der Regierung fordern, Tätigkeitserwartungen erzeugen und Reaktionen der Regierung den Bürgern das Gefühl vermitteln müssen, bei den Amtsinhabern in allen Wechselfällen politischer Entwicklungen gut aufgehoben zu sein.

Um sich der Beantwortung ihrer Frage stringent zu nähern, definiert Bytzek zunächst den Begriff des Ereignisses (S. 49 ff.). Dieses hat drei Eigenschaften: (1) Es ist ein dem po- 
litischen Prozess exogenes, also nicht durch Politiker im Hauptgeschäft hervorgebrachtes Geschehen; (2) es existieren Reaktionserwartungen an die Regierung auf Seiten der Wählerschaft, und (3) es gibt eine ausreichende öffentliche Wahrnehmbarkeit (Medienpräsenz). Nach Anwendung aller Kriterien eignen sich im Untersuchungszeitraum (1977 bis 2003) 43 Ereignisse für die Analyse (siehe die Übersicht auf den Seiten 65 und 66).

Bevor Bytzek zu einer systematischen Analyse übergeht, etabliert sie den unerlässlichen Nexus zwischen Ereignis und individueller Reaktion darauf. Dazu wird ein Modell individuellen Wählerverhaltens entworfen. Der Wirkungszusammenhang zwischen Ereignis und Einschätzung der Regierung in seinem Gefolge wird sodann an zwei Fällen im Zeitverlauf auf der Individualebene getestet. Im Ergebnis dieser vorbereitenden Analyse stellt Bytzek fest, dass der Einfluss symbolischer Politik auf die Regierungseinschätzung nicht endgültig geklärt, dafür aber ein eindeutiger Einfluss der Medieneinschätzung der Regierungsleistung zu konstatieren ist. Außerdem zeigt sich, dass Ereignisse so stark wirken können, dass sie den Wahrnehmungsfilter „Parteiidentifikation“ überwinden (S. 141 bis 143).

Im weiteren Verlauf analysiert Bytzek die Wirkung von Ereignissen auf die aggregierte Regierungseinschätzung. Dazu formuliert sie zunächst Wirkungserwartungen für unterschiedliche Ereignisklassen. Um die Wirkung realistisch testen zu können, modelliert sie die Wirkung der wirtschaftspolitischen Leistung der Regierung (gemessen über die Arbeitslosenquote) als Langfristfaktor und befindet, dass Ereignisse die Wirkung dieses Faktors tatsächlich, wenn auch nur kurzfristig, überformen. Die Regierungspopularität schwingt nach Abklingen der unmittelbaren Ereigniswirkung jedoch nicht vollständig auf das Ausgangsniveau zurück. Vielmehr hinterlassen Ereignisse einen „Memoryeffekt“, das heißt, es gibt eine längerfristige Niveauverschiebung in der Regierungspopularität. Gleichzeitig offenbart die Analyse, dass Ereignisse einen kleinen Regierungspartner deutlich stärker betreffen als die jeweilige große Regierungspartei.

Dies ist das wohl überraschendste Resultat der Studie, deren zentrales Ergebnis darin besteht, dass politische Ereignisse tatsächlich eine den theoretischen Erwartungen entsprechende Wirkung auf die Regierungspopularität haben. Die wichtigste Konsequenz aus der Arbeit für die Wahl- und Wählerforschung muss heißen, dass Ereignisse insbesondere kurz vor Wahlen unbedingt in die Analyse des Wahlverhaltens und in Wahlprognosen einzubeziehen sind.

Bei einer so reflektiert durchgeführten und gut dokumentierten Studie wie der von Bytzek fällt es schwer, kritische Punkte zu finden. Am ehesten hatte der Rezensent Zweifel an Bytzeks Feststellung, dass Ereignisse mit starkem Einfluss den Wahrnehmungsfilter der Parteiidentifikation durchaus überwinden können. Prüffälle sind für sie die CDU-Spendenaffäre, die auch bei den Anhängern von Union und FDP zu Reputationsverlusten der CDU führte sowie der Umgang des ersten Kabinetts Schröder mit dem Kosovokrieg, der auch bei CDU- und FDP-Wählern eine verbesserte Einschätzung der Regierungsleistung bewirkte. Wenn man die Identifikation aber, wie Bytzek es tut, mit Morris P. Fiorina als „running tally“, als laufende Buchführung über politisches Entscheiden betrachtet, ist es nicht plausibel zu erwarten, dass diese so stark wahrnehmungsfilternd wirken könne, dass eine grundsätzlich für gut gehaltene Politik nur deshalb schlechte Bewertungen bekommt, weil sie vom anderen politischen Lager umgesetzt wird oder dass massives Fehlverhalten der „eigenen“ Partei vollständig ausgefiltert werden kann. Der Befund ist daher nicht unerwartet und erweckt zumindest keinen zusätzlichen Zweifel an der analytischen Tauglichkeit des Konzeptes „Parteiidentifikation“. 
Unerfreulich an dem Band ist die Lektorierung. Für zahlreiche Tippfehler sowie unvollständige und übervollständige Sätze ist zwar der Verfasser in erster Linie verantwortlich, aber Herausgeber und Verlag haben ebenso für die formale Genauigkeit ihrer Manuskripte zu bürgen.

Abschließend ist festzuhalten, dass Bytzeks Buch sowohl politischen Praktikern als auch wissenschaftlich Interessierten unbedingt zur Lektüre zu empfehlen ist. Es untersucht systematisch ein bislang von der deutschen Wahlforschung stiefmütterlich behandeltes Phänomen, von dem der Alltags- wie der Medienwelt längst klar ist, dass es erhebliche politische Implikationen hat. Bytzek versteht es, Brücken zu sehr verschiedenen Öffentlichkeiten zu schlagen, indem sie einerseits äußerst sorgfältig ihr methodisches Vorgehen dokumentiert, andererseits ihre Ergebnisse so aufbereitet, dass der methodisch weniger gewandte Praktiker ihre Analyse mit Gewinn lesen und ihre Schlussfolgerungen nachvollziehen kann.

Kai-Uwe Schnapp

\section{Komplettes Handbuch des Bayerischen Landtags 1918 bis 1933}

\section{Lilla, Joachim (Bearbeiter): Der Bayerische Landtag 1918/1919 bis 1933. Wahlvorschläge- Zusammensetzung - Biographien (Materialien zur bayerischen Landesgeschichte, Band 21), Kommission für bayerische Landesgeschichte, München 2008, XLI, 618 Seiten, € 42,-.}

Nach einem Pontifikalamt konstituierte sich am 28. April 1933 der den Schein der Legalität nur notdürftig wahrende "gleichgeschaltete“ Bayerische Landtag. Der Plenarsaal in der Prannerstraße - im Krieg später zerstört - war mit Hakenkreuzfahnen und weißblauen Rautenfahnen ausstaffiert. Im Schnellverfahren verabschiedete der Landtag unter seinem neuen Präsidenten Hermann Esser, einem „alten Mitkämpfer Adolf Hitlers“, am folgenden Tag den Entwurf des „Gesetzes zur Behebung der Not des bayerischen Volkes und Staates“ durch Erheben von den Plätzen. Gegen das „Ermächtigungsgesetz“ stimmte wie zuvor im Reichstag nur die Sozialdemokratische Fraktion: Von den 17 Abgeordneten fehlte „unentschuldigt“ Michael Poeschke, der kurz zuvor „völlig verstört“ (Wilhelm Hoegner, S. 458) aus dem KZ Dachau entlassen worden war. Danach trat der Landtag nicht mehr zusammen. Am 13. November feierte die Nationalsozialistische Fraktion den parlamentarischen Kehraus. Die Sozialdemokraten, deren Mandate am 7. Juli „kassiert“ wurden, hatte der Landtagspräsident bereits am 23. Juni wissen lassen, dass er die zugeteilten Mandate „bis auf weiteres als erloschen“ (S. XXXVI) betrachtete. In Verbindung mit der Auflösung des Reichstags am 14. Oktober 1933 wurden die zuvor gleichgeschalteten „Volksvertretungen“ aufgelöst. 1949, drei Jahre nach dem Wiederbeginn des parlamentarischen Lebens, konnten der Bayerische Landtag und der Bayerische Senat in das renovierte Maximilianeum einziehen, das zu einem Synonym für das Landesparlament geworden ist.

Das Handbuch von Joachim Lilla ist mehr als ein biographisches Nachschlagewerk über die 654 Abgeordneten des Bayerischen Landtages zwischen der Revolution von 1918/19 und dem „Ende der Parteien“ in Deutschland 1933. Neben den Mandatsträgern widmet der Bearbeiter den 988 erfolglosen Wahlbewerbern sein Augenmerk. In der gebotenen Kürze referiert er das Wahlrecht, behandelt die Stimmkreiseinteilung und die bayerische Spezialität der Landesabgeordneten, die - als Instrument gegen die „Atomisierung des politischen Lebens" (Heinrich von Jan, S. XXXI) gepriesen - den im Landtag vertretenen Parteien 